\title{
Evaluation of a possible upgrade of the IAU 2006 precession
}

\author{
J.-C. Liu ${ }^{1}$ and N. Capitaine ${ }^{2}$
}

\author{
1 School of Astronomy and Space Science, Key Laboratory of Modern Astronomy and Astrophysics (Ministry of Education), \\ Nanjing University, 163 XianLin Avenue, 210023 Nanjing, PR China \\ e-mail: jcliu@nju.edu.cn \\ 2 SYRTE, Observatoire de Paris, PSL Research University, CNRS, Sorbonne Universités, UPMC Univ. Paris 06, LNE, \\ 61 avenue de l'Observatoire, 75014 Paris, France \\ e-mail: $n$.capitaine@obspm. fr
}

Received 14 April 2016/ Accepted 8 September 2016

\begin{abstract}
Context. The International Astronomical Union (IAU) adopted a new precession model at its 2006 General Assembly. After more than ten years since the publication of the so-called IAU 2006 precession, we have noticed progress in solar system ephemerides and geophysical observations, which can be used to refine the precession model. Another progress is the increase by $30 \%$ since 2003 , of the length of the very long baseline interferometry (VLBI) observations to be compared with the theoretical model.

Aims. The aim of this paper is to investigate the possibility of upgrading the IAU 2006 precession model based on new solutions of the Earth-Moon barycenter (EMB) motion, new theoretical contributions to the precession rates, and the revised $J_{2}$ long-term variation obtained from the satellite laser ranging (SLR).

Methods. The new precession expressions for the ecliptic are derived by fitting the new analytical planetary theory VSOP2013 to the numerical ephemerides DE422 or INPOP10a. The solution for the precession of the equator was obtained by integrating the dynamical precession equations with the use of an updated Earth model including the $J_{2}$ quadratic long-term variation. The new precession expressions (denoted LC solution in this paper) are compared with the IAU 2006 model by using the most accurate VLBI observations up to 2015.

Results. For the precession of the ecliptic, the changes in the new solutions with respect to the IAU 2006 are about several tens of microarcseconds in the linear terms of $P_{\mathrm{A}}$ and $Q_{\mathrm{A}}$. The upgraded precession of the equator is such that the quadratic and cubic terms in the quantity $\psi_{\mathrm{A}}$ differ significantly from IAU 2006 due to the revised $J_{2}$ model. The statistics of the VLBI celestial pole offsets (1979-2015) and least squares fits with different empirical models, show that the LC precession is slightly more consistent with the VLBI observations, but the improvement relative to the IAU 2006 model is not definitely convincing at present.

Conclusions. The upgraded LC precession obtained with the latest theoretical and observational improvements has shown some advantages with respect to the IAU 2006 model. However, due to negligible changes in the precession of the ecliptic and large uncertainties in the $J_{2}$ variation, we recommend that the standard IAU 2006 model be retained for practical use and continuity reason. The next precession-nutation model should be constructed with a uniform dynamical approach in the framework of ICRF3 and Gaia reference frame and without the use of a conventional ecliptic.
\end{abstract}

Key words. astrometry - celestial mechanics - reference systems - ephemerides

\section{Introduction}

The current standard precession-nutation model recommended by the International Astronomical Union (IAU) and the International Earth Rotation and Reference System Service (IERS) is composed of the IAU 2006 precession (Capitaine et al. 2003a; Hilton et al. 2006, also known as P03 model) and the IAU 2000A nutation series (Mathews et al. 2002) with IAU 2006 adjustments. In order to be most compatible with the IAU $2000 \mathrm{nu}-$ tation and to provide polynomial developments for a number of quantities for use in both equinox based and CIO (Celestial intermediate origin) based celestial-to-terrestrial reference system transformation paradigms, the IAU 2006 precession computations followed the traditional way in which a time-dependent "ecliptic" (i.e., a kind of representation of the mean orbital motion of the Earth-Moon barycenter (EMB) in the Geocentric Celestial Reference System (GCRS)) was used as an intermediate plane for expressing the contributions to the precession rates resulting from the external torques induced by the Sun, the Moon, and the planets. Capitaine et al. (2004) compared the $\mathrm{P} 03$ precession with other post-2003 developments, such as F03 (Fukushima 2003), HF04 (Harada \& Fukushima 2004), B03 (Bretagnon et al. 2003), and W94 (Williams 1994) models, concluding that the $\mathrm{P} 03$ precession of the ecliptic and equator were the most accurate among various solutions. From observational and practical points of view, the $\mathrm{P} 03$ precession model is consistent with the very long baseline interferometry (VLBI) observations, therefore is quite efficient for predicting the secular motion of the Celestial Intermediate Pole (CIP) in the GCRS. These are the reasons why the IAU adopted the P03 solution as the standard IAU 2006 model in the Prague General Assembly.

The IAU 2006 precession of the ecliptic (Capitaine et al. 2003a) was derived by fitting the analytical ephemerides VSOP87 (Bretagnon \& Francou 1988) to the long-term numerical ephemerides DE406 (Standish 1998) over the time span J1000.0 to J3000.0. The best rotations from DE406 equatorial coordinate system to the ecliptic system were found, then the corrections restricted by observational data (namely DE406) were applied to VSOP87 polynomial expressions for the osculating elements $p$ and $q$, which represent the secular motion of the 
moving ecliptic pole in the fixed ecliptic coordinate system of J2000. The final solution was used to replace the IAU 1976 expressions for the precession of the ecliptic (Lieske et al. 1977).

Regarding the precession of the equator, the previous version, the IAU 2000 model (Mathews et al. 2002), consists only of corrections to the precession rates in longitude and obliquity of the IAU 1976 precession. The effect of the changes on the higher-order terms in the precession theory were ignored; thus the IAU 2000 precession was not consistent with the dynamical theory of the Earth's rotation. The IAU 2006 precession of the equator (Capitaine et al. 2003a), in contrast, is dynamically consistent: the basic precession quantities $\psi_{\mathrm{A}}$ and $\omega_{\mathrm{A}}$ were derived by solving the dynamical differential equations using improved ecliptic precession, integration constants provided by IAU 2000 precession with careful consideration of perturbing effects, and the best non-rigid Earth model available at that time. One notable progress of the IAU 2006 precession of the equator is the inclusion of a negative $J_{2}$ rate ( $J_{2}$ is known as the Earth's form factor or the second-degree zonal harmonic of the Earth's gravitational field) that is generally attributed to the postglacial rebound of the Earth's mantle. The value for the $J_{2}$ rate adopted in the IAU 2006 model is

$\frac{\dot{J}_{2}}{J_{2}}=-2.7774 \times 10^{-6} \mathrm{cy}^{-1}$,

corresponding to

$J_{2}=1.0826358 \times 10^{-3}$ and $\dot{J}_{2}=-3.001 \times 10^{-9} \mathrm{cy}^{-1}$.

The contribution of this linear change in $J_{2}$ to the precession rate in longitude, $r_{\psi}$, is of -14 mas $t$, which yields about -7 mas $t^{2}$ in the polynomial expression for $\psi_{\mathrm{A}}$. This is the main reason for the quadratic term difference between the IAU 2006 and IAU 2000 equator precession in longitude. However, the relative uncertainty of the $J_{2}$ rate is about $20 \%$ (Williams 1994), which is the main limiting factor of the accuracy of the precession in longitude. Thereafter Capitaine et al. (2005, denoted P04 paper) tried to improve the $\mathrm{P} 03$ precession of the equator using a refined model for the contribution of non-rigid Earth and revised integration constants from VLBI data. The conclusion was to retain the P03 solution as the replacement for the IAU 2000 model due to the fact that there was no convincing evidence that the P04 would deliver better accuracy than the P03 precession model.

Since the publication of the IAU 2006 precession in 2003, more accurate analytical and numerical ephemerides for the solar system planets, which provide improved EMB motion, have been made available for recalculating the precession of the ecliptic. Moreover, updated theoretical contributions to the precession rates and observations for the Earth's gravitational field enable us to look into the possibility of improving the precession of the equator. This paper reports our effort to develop refined precession solutions based on these scientific advancements.

\section{Improving the precession of the ecliptic}

The ecliptic pole is defined by the mean orbital angular momentum of the Earth-Moon barycenter (EMB) about the Sun (rather than the solar system barycenter, SSB) in the Barycentric Celestial Reference System (BCRS). The precession of the ecliptic is defined as the secular part of the ecliptic pole motion in the initial reference system and is represented by the parameters $P_{\mathrm{A}}$ and $Q_{\mathrm{A}}$ :

$P_{\mathrm{A}}=\sin \pi_{\mathrm{A}} \sin \Pi_{\mathrm{A}} ; \quad Q_{\mathrm{A}}=\sin \pi_{\mathrm{A}} \cos \Pi_{\mathrm{A}}$, where $\pi_{\mathrm{A}}$ is the inclination of the moving ecliptic to the fixed ecliptic, and $\Pi_{\mathrm{A}}$ is the longitude of the node of the moving ecliptic upon the fixed ecliptic of J2000.0. The analytical planetary theories Variations Séculaires des Orbites Planétaires (VSOP, Bretagnon \& Francou 1988) introduced the equinoctial elements $p$ and $q$ for the Earth-Moon barycenter:

$p=\sin \frac{\pi_{\mathrm{A}}}{2} \sin \Pi_{\mathrm{A}} ; \quad q=\sin \frac{\pi_{\mathrm{A}}}{2} \cos \Pi_{\mathrm{A}}$.

The rigorous relations between $P_{\mathrm{A}}, Q_{\mathrm{A}}$, and $p, q$ are such that:

$$
\begin{gathered}
P_{\mathrm{A}}=2 p \sqrt{1-p^{2}-q^{2}} ; \quad Q_{\mathrm{A}}=2 q \sqrt{1-p^{2}-q^{2}} \\
p=\frac{P_{\mathrm{A}}}{2 \sqrt{1-P_{\mathrm{A}}^{2}-Q_{\mathrm{A}}^{2}}} ; \quad q=\frac{Q_{\mathrm{A}}}{2 \sqrt{1-P_{\mathrm{A}}^{2}-Q_{\mathrm{A}}^{2}}} .
\end{gathered}
$$

\subsection{The analytical planetary theory VSOP 2013 and the numerical ephemerides DE422 INPOP10a}

Since the IAU 2006 precession of the ecliptic had been derived using VSOP87 (Bretagnon \& Francou 1988) and DE406 (Standish 1998), progress in the solutions for the EMB motion have been made. The new analytical planetary theory VSOP2013, together with the Theory of the Outer Planets TOP2013 were developed by Simon et al. (2013). The VSOP2013 is fitted to the INPOP10a (Intégrateur Numérique Planétaire de l'Observatoire de Paris) numerical planetary ephemeris (Fienga et al. 2011) and the accuracy between 1890 and 2000 is improved by a factor two to 24 depending on the planets, as compared to its previous version VSOP2000. The long-term data (from -4000 to 8000 , used in this study) is more accurate by a factor of five with respect to VSOP2000. Since the VSOP2000 solution was ten to 100 times more precise than the VSOP87 as used in the IAU 2006 model (Simon et al. 2013), the improvement of VSOP2013 with respect to VSOP87 is quite remarkable. The VSOP2013 has provided numerical Chebyshev Ephemerides from -4500 to 4500 with a similar structure to DE ephemerides (Jet Propulsion Laboratory Development Ephemeris). It also provides users with the elliptic elements, including $p$ and $q$, for the eight planets in the form of mixed series which contain secular, Fourier, and Poisson terms. The secular parts (polynomials up to the eighth degree in $t$ ) of $p$ and $q$ for EMB motion representing the precession of the ecliptic will be studied in the following.

The JPL DE422 numerical ephemerides (Folkner et al. 2008), covering the time interval between JD 0625648.5 (December 7, 3001 BC) and JD 2816816.5 (January 30, 3000 AD), was created in 2009. It is referred to the International Celestial Reference Frame (ICRF) and includes nutations and liberations. DE422 has made use of new observations including ranging data from Mars spacecrafts, the effects of nutation and libration of the Moon, Venus Express (VEX) ranging data for the improvement of Venus orbit, and Cassini data for better constraints on Jupiter and Saturn orbits. All these data improved the estimation of EMB orbit, the strongest constraints being given by the ranging data from Mars spacecraft.

The numerical ephemerides INPOP10a, also in the framework of the ICRF, was developed by Fienga et al. (2011) and was released in 2010. As compared to the previous INPOP versions, several improvements have been introduced in the fitting process, the data sets used in the fit, and the selection of fitted parameters. More than 136000 planetary observations including the latest Messenger, Mars Express, Venus Express, and Cassini 
J.-C. Liu and N. Capitaine: Upgrade of the IAU precession model

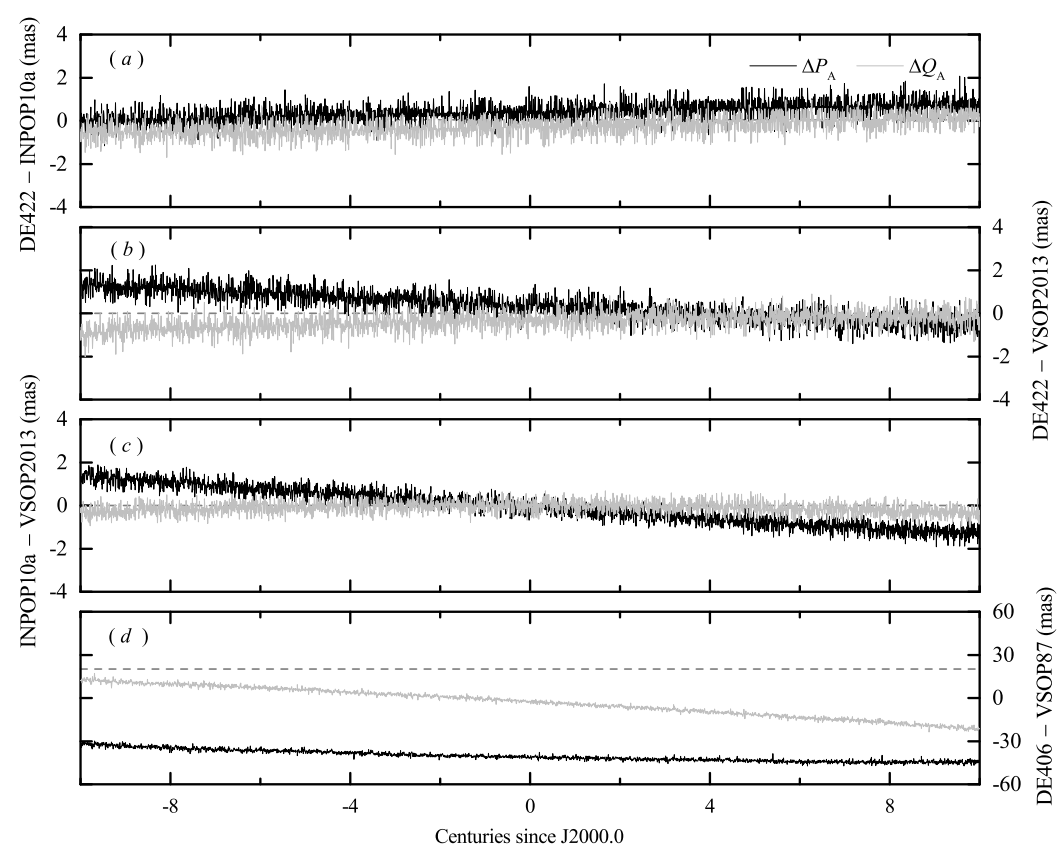

Fig. 1. Difference of $P_{\mathrm{A}}$ and $Q_{\mathrm{A}}$ between various ephemerides used in this paper over 20 centuries (1000-3000). A different scale is used in the bottom plot (d).

data, and lunar laser ranging (LLR) normal points have been applied for the construction of INPOP. The main characteristics are the fit of the product, $G M_{\odot}$, of the solar mass with the gravitational constant instead of the astronomical unit, and the determinations of the post-Newtonian (PPN) parameters as well as adjustments of the solar oblateness and of asteroid masses. The new IAU resolution about the astronomical unit (AU, Capitaine et al. 2011) was implemented for the first time in INPOP10a, together with the best estimations of the mass of planets provided by the IAU 2009 solution (Luzum et al. 2011). Statistics of planetary post residuals after fit show that the accuracy of the INPOP10a has been largely improved in comparison to the previous version INPOP08 (Fienga et al. 2010).

\subsection{Revision of the ecliptic precession based on new developments}

In this subsection we show the possibility of improving the ecliptic precession by fitting VSOP2013 theory to DE422/INPOP10a: the later ephemerides are introduced as additional constrains. The purpose of using both modern numerical ephemerides prolongated over the same time span (1000-3000) is to investigate the effect of different ephemerides on the estimation of the ecliptic precession.

For VSOP2013, $P_{\mathrm{A}}$ and $Q_{\mathrm{A}}$ are obtained directly using elliptic elements $p, q$ with Eq. (5). For DE422 or INPOP10a, we first interrogate the ephemerides to obtain the EMB heliocentric position $\boldsymbol{r}$ and velocity $\boldsymbol{r}$ in the International Celestial Reference System (ICRS, i.e., the equatorial coordinate system) and calculate the angular momentum:

$\boldsymbol{h}=\boldsymbol{r} \times \dot{\boldsymbol{r}}$.

In the next step, the components of the angular momentum $\boldsymbol{h}$ are transformed from the ICRS to the J2000 ecliptic frame using the following equation:

$\boldsymbol{h}^{\mathrm{ecl}}=\mathcal{R}_{3}\left(\psi_{0}\right) \mathcal{R}_{1}\left(\epsilon_{0}\right) \mathcal{R}_{3}\left(-\phi_{0}\right) \cdot \boldsymbol{h}$,

where the geometrical definition of the angles $\psi_{0}, \epsilon_{0}$, and $\phi_{0}$ are given in Fig. 1 of Capitaine et al. (2003a). Initially, the values are provided by VSOP2013 (Simon et al. 2013) as

$\psi_{0}=0, \quad \epsilon_{0}=84381^{\prime \prime} .41136, \quad \phi_{0}=-0$ '”05188.

At last, the parameters $P_{\mathrm{A}}$ and $Q_{\mathrm{A}}$ for DE422 and INPOP10a can be derived from the vector $\boldsymbol{h}^{\mathrm{ecl}}=\left(h_{x}^{\mathrm{ecl}}, h_{y}^{\mathrm{ecl}}, h_{z}^{\mathrm{ecl}}\right)^{\mathrm{T}}$ in the ecliptic coordinate system:

$P_{\mathrm{A}}=\frac{h_{x}^{\mathrm{ecl}}}{\left|\boldsymbol{h}^{\mathrm{ecl}}\right|}, \quad Q_{\mathrm{A}}=-\frac{h_{y}^{\mathrm{ecl}}}{\left|\boldsymbol{h}^{\mathrm{ecl}}\right|}$,

considering that $P_{\mathrm{A}}$ and $Q_{\mathrm{A}}$ are the $x$ and $-y$ components of the moving ecliptic pole vector in the fixed ecliptic at J2000. $\left|\boldsymbol{h}^{\mathrm{ecl}}\right|$ is the norm of the vector $\boldsymbol{h}^{\text {ecl }}$.

Figure 1 (panels a-c) shows the difference between DE422, INPOP10a, and VSOP2013 for the EMB motion represented by $P_{\mathrm{A}}$ and $Q_{\mathrm{A}}$ in the dynamical ecliptic frame over 20 centuries, while the bottom panel (d) is the same plot for the difference [DE406 - VSOP87] which were studied in the IAU 2006 model (see similar Figs. 2 and 4 of Capitaine et al. 2003a). The thickness of the curves is due to the short periodic terms; the slope and curvature are attributed to the misalignment of the secular part; the overall offset of the curves is due the imperfection of the transformation angles between the ecliptic and equatorial coordinate system. For the last panel, original transformation angles in the P03 paper $\left(\psi_{0}=0, \epsilon_{0}=84381\right.$ ". 4 , and $\left.\phi_{0}=0\right)$ are applied, which cause large offsets between $P_{\mathrm{A}}$ and $Q_{\mathrm{A}}$. Panel d in Fig. 1 clearly displays that the secular discrepancy [DE422 - VSOP2013] or [INPOP10a - VSOP2013] is significantly less than [DE406 - VSOP87] because the VSOP87 analytical theory was fitted to the DE200 ephemerides (constructed in 1981), and thus was not up-to-date relative to DE406 (constructed in 1995) at the time of developing the IAU 2006 precession.

The situation has now changed: the more recent ephemerides VSOP2013, INPOP10a, and DE422 have the same level of accuracy, therefore, in principle, either VSOP2013, INPOP10a, or DE422 can be used to construct the precession of the ecliptic independently. However, using only numerical ephemerides could 
Table 1. Equator to Ecliptic coordinate system transformation angles for DE422 and INPOP10a.

\begin{tabular}{l|llr}
\hline \hline & DE422 & INPOP10a & Uncertainty \\
\hline$\phi_{0}$ & $0 \prime \prime 051033$ & $0 \prime \prime 052032$ & $22 \mu \mathrm{as}$ \\
$\epsilon_{0}-84381^{\prime \prime}$ & $0 \prime \prime 411063$ & $0 \prime \prime 411365$ & $8 \mu \mathrm{as}$ \\
$\psi_{0}$ & $0^{\prime \prime} \cdot 112513$ & $0^{\prime \prime} 113602$ & $20 \mu \mathrm{as}$ \\
\hline
\end{tabular}

bring a risk of contamination by long periodic terms. Capitaine et al. (2004) compared various precession models of the ecliptic and found that the F03 (Fukushima 2003) and HF04 (Harada \& Fukushima 2004) models fitted to DE405 over hundreds of years seriously deviate from observations because they are largely affected by residual contributions of long period terms that have been incorrectly dealt with. On the other hand, the IAU 2006 (Capitaine et al. 2003a) model avoided this problem without any bias through a fit of VSOP87 to DE406 in the interval 1000-3000. Here, we follow a similar approach by a fit of VSOP2013 to DE422/INPOP10a in order to obtain the final secular part of the EMB motion.

The 250-day sampling series of $(t, \Delta p, \Delta q)$ in the sense of [DE422/INPOP10a - VSOP2013] between J1000 and J3000 are fitted to the fifth order polynomials. The resulting coefficients of constant terms $p_{0}$ and $q_{0}$ are used to improve the rotation angles, while the coefficients of $t^{1}-t^{5}$ terms are added to corresponding secular terms given by VSOP2013. Only two iterations are required to reach the converged results. The final rotation angles from equatorial to ecliptic coordinate systems for DE422 and INPOP10a are presented in Table 1. The total corrections, along with their uncertainties to be added to the secular variations of $p$ and $q$ (except for the constant terms), are given in Table 2, where the numerical coefficients are in microarcseconds ( $\mu$ as), and $t$ is measured in Julian centuries elapsed from J2000.0. $P_{\mathrm{A}}$ and $Q_{\mathrm{A}}$ are transformed from $p$ and $q$ discrete points using Eq. (5) and then fitted to the fifth order polynomial to derive the coefficients from $t^{1}$ to $t^{5}$. Table 3 gives the updated ecliptic precession quantities $P_{\mathrm{A}}$ and $Q_{\mathrm{A}}$ corresponding to the values in Table 2 . In this way, the real uncertainties of the $P_{\mathrm{A}}$ and $Q_{\mathrm{A}}$ polynomial coefficients are not easy to derive: they should depend on the errors in the used ephemerides. Since $P_{\mathrm{A}} / Q_{\mathrm{A}}$ and $p / q$ are equivalent, the error of $t^{1}$ coefficients of $P_{\mathrm{A}}$ and $Q_{\mathrm{A}}$ should not exceed several microarcseconds per year, while the errors in higher order terms are too small to be considered. The above Tables 2 and 3 illustrate the following points:

- in Table 2, the difference between linear terms of $p$ and $q$ between DE422, INPOP10a, and VSOP2013 are at the order of several tens of microarcseconds, while higher order terms are negligible;

- from both tables, we found that the VSOP2013 EMB secular motion is closer to DE422 than to INPOP10a, although VSOP2013 was nominally fitted to INPOP10a;

- the discrepancy of the solution for the precession of the ecliptic $P_{\mathrm{A}}$ and $Q_{\mathrm{A}}$ in the present work and IAU 2006 model is several tens of microarcseconds for linear term and about $10 \mu$ as for square term.

- in fact, the difference in precession of the ecliptic between our new solution and the IAU model is about two orders of magnitudes lower than [IAU 2006 - other post-2003 solution] which shows good agreement between current results and the IAU 2006 model. Here "post-2003 solutions" refer to F03 (Fukushima 2003), HF04 (Harada \& Fukushima 2004), B03 (Bretagnon et al. 2003), and W94 (Williams 1994) solutions.
According to the comparative study in Capitaine (2004), a difference in the $t$ term of $P_{\mathrm{A}}$ and/or $Q_{\mathrm{A}}$ is fully reflected in the coefficients for $\epsilon_{\mathrm{A}}$ and/or $\chi_{\mathrm{A}}$ expressions, both referring directly to the ecliptic. On the other hand, the largest effect in the basic quantities for the precession of the equator, $\psi_{\mathrm{A}}$ and $\omega_{\mathrm{A}}$ expressions, is of the order of 0.1 mas in the $t^{2}$ term for a difference of a few mas in the $t$ term of $P_{\mathrm{A}}$ and/or $Q_{\mathrm{A}}$. This means that the changes given by Table 3 would give effects of the order of tens of $\mu$ as in the $t$ term of $\epsilon_{\mathrm{A}}$ and/or $\chi_{\mathrm{A}}$, and of only about of $1 \mu$ as in the $t^{2}$ terms of $\psi_{\mathrm{A}}$ and $\omega_{\mathrm{A}}$. This order of effect is not crucial for deriving the precession of the equator as compared to the improved theoretical contributions, especially that due to the updated $J_{2}$ variation (see Sect. 3.1). The above conclusions have been verified by testing both ecliptic precession for DE422 and INPOP10a: very similar expressions for the precession of the equator are obtained. Given that small difference, we will provide in the following sections, only the solution for the precession of the equator corresponding the DE422-derived ecliptic precession (cf. lines 1 and 3 of Table 3 ).

\section{Improving the precession of the equator based on recent progress}

The solutions for the precession of the equator are derived by solving the differential equations given in Williams (1994) or Capitaine et al. (2003a):

$\sin \omega_{\mathrm{A}} \frac{\mathrm{d} \psi_{\mathrm{A}}}{\mathrm{d} t}=\left(r_{\psi} \sin \epsilon_{\mathrm{A}}\right) \cos \chi_{\mathrm{A}}-r_{\epsilon} \sin \chi_{\mathrm{A}}$
$\frac{\mathrm{d} \omega_{\mathrm{A}}}{\mathrm{d} t}=r_{\epsilon} \cos \chi_{\mathrm{A}}+\left(r_{\psi} \sin \epsilon_{\mathrm{A}}\right) \sin \chi_{\mathrm{A}}$.

The classical precession quantities $\psi_{\mathrm{A}}$ and $\omega_{\mathrm{A}}$ are chosen as primary parameters because they can be regarded as the angular coordinates of the CIP (or moving equator) with respect to the fixed J2000 ecliptic and the specified precession-rate adjustments can be applied directly and unambiguously.

The above equations transform the precession rates of the mean celestial pole in longitude and obliquity $\left(r_{\psi} \sin \epsilon_{\mathrm{A}}\right.$ and $r_{\epsilon}$ ) from the moving ecliptic system to the fixed ecliptic system through the angle $\chi_{\mathrm{A}}$, which is called planetary precession measured along the moving equator between the intersections of the moving ecliptic and the fixed ecliptic ${ }^{1}$. The other two equations for precession quantities $p_{\mathrm{A}}$ (general precession) and $\epsilon_{\mathrm{A}}$ (obliquity of date) are solved simultaneously with the geometrical relation

$\sin \chi_{\mathrm{A}} \sin \omega_{\mathrm{A}}=P_{\mathrm{A}} \cos p_{\mathrm{A}}+Q_{\mathrm{A}} \sin p_{\mathrm{A}}$.

The resolution of the above equations for computing the IAU 2006 precession was based on the use of a semi-analytical method (with GREGOIRE software, see description in the appendix of Capitaine et al. 2003b). In the present work, we follow a universal numerical approach that has been verified to give the same results for the IAU 2006 case. First, the classical 7(8)-degree Runge-Kutta-Fehlberg (RKF) method ${ }^{2}$ was employed to derive the discrete points (250-day step over $2000 \mathrm{yr}$ centered on $\mathrm{J} 2000.0$ ) for basic quantities $\psi_{\mathrm{A}}, \omega_{\mathrm{A}}$ and secondary quantities $\epsilon_{\mathrm{A}}, \chi_{\mathrm{A}}$, and $p_{\mathrm{A}}$, then the fifth order polynomial expressions for these quantities are obtained by an unweighted least squares fit.

\footnotetext{
$\chi_{\mathrm{A}}$ is also known as the precession of the ecliptic.

2 RKF is a single step integrator for the numerical solution of ordinary differential equations. RKF7(8) method is of order $O\left(h^{7}\right)$ with an error
} estimator of order $O\left(h^{8}\right)$, where $h$ is length of step. 
Table 2. Corrections $\Delta p$ and $\Delta q$ to VSOP2013 secular terms.

\begin{tabular}{lrrrrrr}
\hline \hline & & $t^{1}$ & $t^{2}$ & $t^{3}$ & $t^{4}$ & $t^{5}$ \\
\hline & $\Delta p$ & -38 & +0.5 & +0.08 & -0.002 & +0.0001 \\
DE422-VSOP2013 & \pm & 2 & 0.3 & 0.04 & 0.003 & 0.00005 \\
\cline { 2 - 7 } & $\Delta q$ & +20 & -1 & -0.5 & +0.003 & +0.0001 \\
& \pm & 2 & 0.2 & 0.06 & 0.002 & 0.00005 \\
\hline \multirow{4}{*}{ INPOP10a-VSOP2013 } & $\Delta p$ & -59 & +0.04 & +0.06 & +0.002 & +0.0002 \\
& \pm & 2 & 0.03 & 0.04 & 0.002 & 0.00005 \\
\cline { 2 - 7 } & $\Delta q$ & +3 & -2 & -0.5 & -0.003 & +0.0007 \\
& \pm & 2 & 0.3 & 0.07 & 0.003 & 0.00006 \\
\hline
\end{tabular}

Notes. The unit is $\mu$ as.

Table 3. Precession quantities of the ecliptic derived from VSOP2013 and DE422/INPOP10a ephemerides and comparison with the IAU 2006 model.

\begin{tabular}{lclccccc}
\hline \hline & & Unit & $t^{1}$ & $t^{2}$ & $t^{3}$ & $t^{4}$ & $t^{5}$ \\
\hline \multirow{4}{*}{ Fit to DE422 } & $P_{\mathrm{A}}$ & $\prime \prime$ & 4.19903 & 0.19401 & $-2.23533 \times 10^{-4}$ & $-1.03944 \times 10^{-6}$ & $2.15694 \times 10^{-9}$ \\
& $\Delta P_{\mathrm{A}}$ & $\mu$ as & -65 & 18 & 1 & -0.1 & 0.01 \\
\cline { 2 - 8 } & $Q_{\mathrm{A}}$ & $\prime \prime$ & -46.81099 & 0.05102 & $5.21368 \times 10^{-4}$ & $-5.5808 \times 10^{-7}$ & $-1.2059 \times 10^{-9}$ \\
& $\Delta Q_{\mathrm{A}}$ & $\mu$ as & 28 & -11 & -3 & 0.1 & 0.02 \\
\hline \multirow{4}{*}{ Fit to INPOP10a } & $P_{\mathrm{A}}$ & $\prime \prime$ & 4.19906 & 0.19400 & $-2.23575 \times 10^{-4}$ & $-1.02313 \times 10^{-6}$ & $2.26088 \times 10^{-9}$ \\
& $\Delta P_{\mathrm{A}}$ & $\mu$ as & -27 & 17 & 1 & -0.1 & -0.001 \\
\cline { 2 - 7 } & $Q_{\mathrm{A}}$ & $\prime \prime$ & -46.81102 & 0.05102 & $5.21248 \times 10^{-4}$ & $-5.64478 \times 10^{-7}$ & $5.5757 \times 10^{-10}$ \\
& $\Delta Q_{\mathrm{A}}$ & $\mu$ as & -10 & -12 & -3 & 0.1 & 0.02 \\
\hline
\end{tabular}

Notes. $\Delta P_{\mathrm{A}}$ and $\Delta Q_{\mathrm{A}}$ are calculated in sense of [New model - IAU 2006].

In order that the refined solution for the precession of the equator be "dynamically consistent" and compliant with the IAU 2000 nutation, we proceed with our computation in the same way as IAU 2006. This means solving the above differential Eq. (10) with the use of (1) the best available expressions for $P_{\mathrm{A}}$ and $Q_{\mathrm{A}}$ for the precession of the ecliptic; (2) theoretical corrections to precession rates; (3) the best available integration constants $r_{0}$ and $u_{0}$ at $\mathbf{J} 2000.0$. The first subject relating to the ecliptic motion has been studied in Sect. 2, so in Sects. 3.1 and 3.2 we investigate the possible improvements in the latter two aspects, respectively, and give the final expression in Sect. 3.3.

\subsection{Upgraded contributions to the precession rates}

The theoretical expressions for the precession rates with respect to the moving ecliptic can be written as:

$r_{\psi}=r_{0}+r_{1} t+r_{2} t^{2}+r_{3} t^{3}+\cdots$

$r_{\epsilon}=u_{0}+u_{1} t+u_{2} t^{2}+u_{3} t^{3}+\cdots$

where the complete list for the theoretical contributions are provided in Table 3 of Capitaine et al. (2003a). The new progress in precession rates since 2003, within our knowledge, includes the following items:

i. Post-Newtonian treatment of the Earth's rotation in the framework of General Relativity (Gerlach \& Klioner 2013). The geodesic precession-induced torque is taken into account to obtain the Euler angles of rigid Earth $(\phi, \psi, \omega$, the later two correspond to precession quantities in longitude and obliquity, while $\phi$ is related to the rotation rate around the pole) in the kinematically non-rotating GCRS. The discrepancies between the SMART solution (Bretagnon et al. 1997) and corrected solutions for the Euler angles are shown to be Poisson-like and the amplitudes after one century are about $200 \mu$ as in $\psi$ and $100 \mu$ as in $\omega$ (see Fig. 2 of quoted paper). The slope of the discrepancy is not clearly visible from the plots and not provided by the authors, therefore this effect was not considered in our computation.

ii. Determination of the $J_{2}$ long-term variation based on satellite laser ranging (Cheng et al. 2013). This will be discussed in detail in the following.

iii. The contribution of tidal Poisson terms on non-rigid Earth rotation (Folgueira et al. 2007). The Poisson terms in the tidal potential generated by solar system bodies contributes $88 \mu \mathrm{as} \mathrm{cy}^{-1}$ to the precession rate in obliquity. It is useful to mention that the largest contribution to nutation in longitude, although not used directly in the integration, is $6 \mu$ as for $2 l^{\prime}-2 F+2 D-2 \Omega$ term.

iv. The effect of second-order torque on precession rates (Lambert \& Mathews 2006, 2008). The contributions to precession rates originating from Elastic Earth, Anelasticity, and Ocean Tides (EL+AE+OT) were found to be $-124 \mu \mathrm{as} \mathrm{cy}^{-1}$ in longitude and $+1844 \mu \mathrm{as} \mathrm{cy}^{-1}$ in obliquity. These should be used to replace the Mathews' non-linear terms in longitude $-960 \mu \mathrm{as} \mathrm{cy}^{-1}$ and in obliquity $+340 \mu \mathrm{as} \mathrm{cy}^{-1}$ (Capitaine et al. 2004). See discussion in Sect. 3.2.

v. The effect from Galactic aberration (Liu et al. 2012). The Galactic aberration in proper motions is caused by the acceleration of the solar system barycenter (SSB) toward the Galactic center, which induces global rotation of the ICRS depending on the distribution of sources. The systematic effect in precession rates caused by the rotation of the reference system is at the order of $10 \mu \mathrm{as} \mathrm{cy}^{-1}$.

The new theoretical contributions ii. to v. will be taken into account in the integration of the precession equations. 
Table 4. Values of $J_{2}$ rate estimated by observations or adopted in the IAU 2006 precession.

\begin{tabular}{lr}
\hline \hline$\dot{J}_{2}\left(10^{-9} \mathrm{cy}^{-1}\right)$ & Reference \\
\hline-3.0 & Yoder et al. (1983) \\
-2.6 & Nerem et al. (1993) \\
$-3.0 \pm 0.5$ & Stephenson \& Morrison (1995) \\
-3.001 & adopted in the IAU 2006 precession \\
\hline
\end{tabular}

\section{$J_{2}$ long-term variation observed by SLR}

It is widely understood that long time changes in $J_{2}$ contribute to the precession of the equator (Williams 1994). Therefore, including the effect of the $J_{2}$ rate in the development for the precession of the equator is an important progress of the IAU 2006 model as compared to the IAU 2000 (Mathews et al. 2002) and most other precession models (F03, Fukushima 2003; B03, Bretagnon et al. 2003). However, the complexity of the $J_{2}$ variation and significant errors in determination of $\dot{J}_{2}$ is the greatest source of uncertainty in the IAU 2006 precession: the accuracy of the precession theory is limited to about $1.5 \mathrm{mas} \mathrm{cy}^{-1}$ (Bourda \& Capitaine 2004; Capitaine et al. 2009).

Generally the long-term trend in $J_{2}$ has been approximated by a negative linear drift attributed to postglacial rebound of the Earth's mantal or the ongoing global isostatic adjustment. Cheng \& Tapley (2004) found from 28-year SLR observational data (1976-2004) a secular decrease of $J_{2}$ at a rate of $(-2.7 \pm 0.4) \times$ $10^{-9} \mathrm{cy}^{-1}$, which is close to the value used in the IAU model (cf. Eq. (2)). Other previous determinations of $J_{2}$ rate have also given similar results within the uncertainties as shown in Table 4.

More recently, Cheng et al. (2013) reported the updated feature in long-term variation in the Earth's oblateness based on the time series of 30-day SLR (satellite laser ranging) estimate of $J_{2}$ between 1976 and 2012. The original estimates of the $C_{20}$ harmonic coefficient of the geopotential are provided in the literature from which we can obtain the series of $J_{2}$ by the simple relation

$J_{2}=-\sqrt{5} C_{20}$

Figure 2 shows the variation of $J_{2}$ relative to its mean value of $\bar{J}_{2}=0.0010826359797$. The number of digits is that of the original data for $C_{20}$. Straight lines and parabola are used as empirical models to interpret the long-term signals in the observational data. Weighted fits of straight lines to the $J_{2}$ series in the interval of 1976-1996 and 1976-2012 are carried out, and the results are shown in Table 5. The estimated linear trend with the data earlier than 1996 (blue solid line in Fig. 2) is $(-3.04 \pm 0.32) \times 10^{-9} \mathrm{cy}^{-1}$, which is in good agreement with other determinations and IAU adopted value, but a much smaller value $(-0.67 \pm 0.19) \times 10^{-9} \mathrm{cy}^{-1}$ for the linear trend can be found if more recent data between 1996 and 2012 are involved in the least squares fit (red solid line in Fig. 2). This fact demonstrates that the deceleration in $J_{2}$ variation is significant, as this can be clearly seen on the plot ( $J_{2}$ value begins to increase during the past $20 \mathrm{yr}$ ). The most important conclusion is that the long-term variation of the Earth's dynamical formfactor $J_{2}$ appears, from SLR observations up to now, to be more quadratic than linear in nature (Cheng et al. 2013). It is therefore more reasonable to describe the $J_{2}$ variation by a parabola (black curve in Fig. 2) for which the fitted coefficients are given in the last line of Table 5. We note that the time interval of observational data (1976.04-2011.93) is slightly longer than that in the

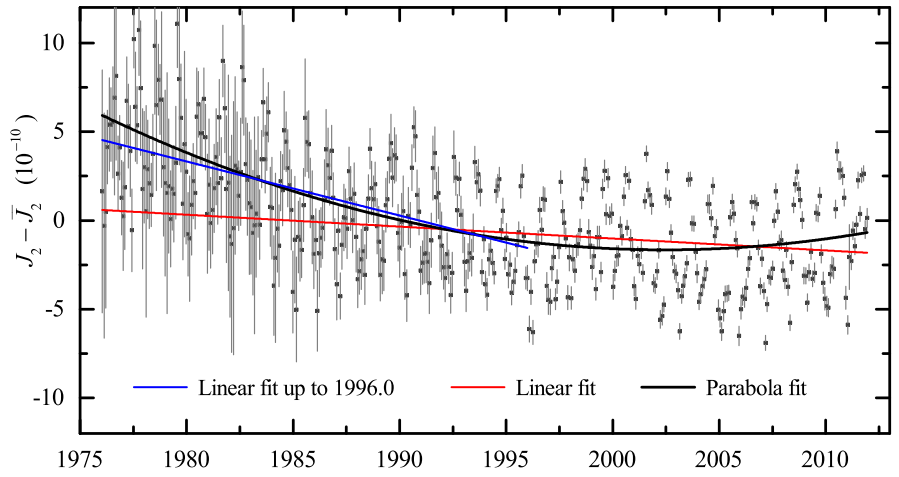

Fig. 2. 30-day estimates of the Earth's $J_{2}$ values from satellite laser ranging (SLR) and its long term variation. The constant $\bar{J}_{2}$ is the mean value for $J_{2}$, which equals 0.0010826359797 . The original data are provided by Cheng et al. (2013). The error bars are shown in gray on the plot.

Cheng et al. (2013) paper (January 1976 to May 2011); however, the numerical values for the coefficients in Table 5 are consistent with Cheng et al. (2013) within the fitted error bars.

It is important to mention that the $J_{2}$ variation is a geophysical effect that cannot be predicted precisely by theories: only empirical models depending on observational data can be applied. Because of its important role in developing the precession model, we use the more realistic quadratic model of $J_{2}$ variation to replace the classical linear model. The contribution of $J_{2}$ variation to the precession rate in longitude ( $r_{\psi}$ in Eq. (12)) is listed in Table 6, where the deceleration term was not considered in the IAU solution. We mention that the $J_{2}$ variation has no effect on the precession rate $r_{\epsilon}$ in obliquity.

\subsection{Integration constants}

The integration constants $r_{0}$ and $u_{0}$ at $\mathbf{J} 2000.0$ for precession rates in longitude and obliquity are crucial for solving the precession Eqs. (10) as they will be exactly the $t^{1}$ terms $\psi_{1}$ and $\omega_{1}$ in the final polynomials for the basic quantities $\psi_{\mathrm{A}}$ and $\omega_{\mathrm{A}}$. Following the strategies of the P03 and P04 paper, we start to calculate the integration constants from the MHB2000 (Mathews et al. 2002) values for the corrections to the precession rates of IAU 1976.

In the MHB model, the corrections to the precession rates are derived from the VLBI fit of theoretical expressions for the precession rate and complex amplitude of a large amount of nutation terms to their observational estimates (or "input data"). In the original MHB2000 paper, the non-linear term $P^{(\mathrm{nr})}$ is $-21050 \mu \mathrm{as} \mathrm{cy}^{-1}$, introducing the correction in precession rate (we note that there is small inconsistency for the value between paragraph [70] and Table 7 in MHB2000 paper):

$\mathrm{d}_{\mathrm{MHB}} \psi_{1}=-299650 \pm 400 \mu \mathrm{as} \mathrm{cy}^{-1}$,

and this value was adopted by the IAU 2006 model. Then the precession corrections used in the P04 model given in Capitaine et al. (2005) are compatible with the updated non-linear terms of $-960 \mu \mathrm{ascy}^{-1}$ in longitude and $+340 \mu \mathrm{ascy}^{-1}$ in obliquity, which read

$\mathrm{d}_{\mathrm{MHB}} \psi_{1}=-299110 \pm 710 \mu \mathrm{as} \mathrm{cy}^{-1}$,

and the P04 model is based on this revised value of $\mathrm{d}_{\mathrm{MHB}} \psi_{1}$.

According to Lambert \& Mathews (2008) the precession corrections should, in principle, be consistent with the updated non-linear terms, that is, EL+AE+OT contributions of 
Table 5. Weighted fits of straight lines and parabola to the $J_{2}$ data from SLR.

\begin{tabular}{rlccc}
\hline \hline & Time interval & $t^{0}$ & $t^{1}$ & $t^{2}$ \\
\hline Linear fit & $1976-1996$ & $1.08263570 \times 10^{-3}$ & $(-3.0 \pm 0.3) \times 10^{-9}$ & - \\
Linear fit & $1976-2012$ & $1.08263587 \times 10^{-3}$ & $(-0.7 \pm 0.2) \times 10^{-9}$ & - \\
Parabola fit & $1976-2012$ & $1.08263582 \times 10^{-3}$ & $(-0.5 \pm 0.2) \times 10^{-9}$ & $(1.1 \pm 0.2) \times 10^{-8}$ \\
\hline
\end{tabular}

Notes. $t$ is measured in Julian centuries centered on J2000.0.

Table 6. The polynomial expressions for the $J_{2}$ contribution refer to Eq. (12).

\begin{tabular}{|c|c|c|c|}
\hline & & $t^{2}$ & $t^{3}$ \\
\hline IAU 2006 & $r_{\psi}\left(J_{2}\right)$ & $-14 \pm 3$ & 0 \\
\hline This paper & $r_{\psi}\left(J_{2}\right)$ & $-2.482 \pm 1$ & $+50.629 \pm 9$ \\
\hline
\end{tabular}

Notes. The unit is mas.

$-124 \mu \mathrm{ascy}^{-1}$ in longitude and $+1844 \mu \mathrm{ascy}^{-1}$ in obliquity (item iv in Sect. 3.1). It requires a re-analysis of the MHB original VLBI data in MHB approach, which provide Basic Earth Parameters (BEPs) including the dynamical flattening $H_{\mathrm{d}}$ to give out the corrected precession rate (Herring et al. 2002). Since the detailed VLBI fits are far beyond the scope of our paper, we decide to keep the value in Eq. (15). This would be reasonable with no precision loss for the following two reasons: (1) the magnitude changes in the non-linear terms from P04 to this paper is about 25 times less than the magnitude from MHB to $\mathrm{P} 04$, which means that the change in the precession rate (relative to P04 value) would be much smaller for the current case (if the relation between the non-linear term and precession rate correction is rectilinear, the resulting value corresponding the new non-linear term is $\mathrm{d}_{\mathrm{MHB}} \psi_{1}=-299088 \mu \mathrm{as} \mathrm{cy}^{-1}$, which is very close to Eq. (15) within the $1 \sigma$ uncertainty: this kind of estimate is just a test and will not be used); (2) we note that from the MHB to the $\mathrm{P} 04$ model, the precession corrections are consistent to within their standard errors although the difference between respective non-linear terms amounts to a considerable value of $20100 \mu \mathrm{ascy}^{-1}$. Therefore it is safe and reasonable to use Eq. (15) as the MHB correction to the precession rate: this keeps our solution dynamically consistent with the MHB and IAU 2006 models. The new MHB integration constants thus used in our calculation are such that

$$
\begin{aligned}
& \left(r_{0}\right)_{\mathrm{MHB}}=5038^{\prime \prime} .7784-0^{\prime \prime} .299110=5038^{\prime \prime} .479290 \mathrm{cy}^{-1} \\
& \left(u_{0}\right)_{\mathrm{MHB}}=0-0^{\prime \prime} .025240=-0^{\prime \prime} .025240 \mathrm{cy}^{-1} .
\end{aligned}
$$

The later expression for $u_{0}$ is exactly the same as that in the IAU 2000 model (Mathews et al. 2002). The new non-linear contribution to the precession rates in longitude and obliquity in Lambert \& Mathews (2008) will be applied as the theoretical contribution in the integration.

The non-ignorable spurious contributions to the MHB precession rates at J2000.0 were estimated in the IAU 2006 paper for the first time, including the "observed" effect resulting from the dependence of the VLBI observed precession rate in longitude on the chosen ecliptic (or the obliquity of epoch):

$\mathrm{d}_{1} \psi_{1}=-2366 \mu \mathrm{as} \mathrm{cy}^{-1}$,

and the effect of non-rigorous treatment of frame bias before 2003:

$\mathrm{d}_{2} \psi_{1}=-384 \mu \mathrm{ascy}^{-1} ; \quad \mathrm{d}_{2} \omega_{1}=+514 \mu \mathrm{as} \mathrm{cy}^{-1}$.
In the original P03 paper, $\mathrm{d}_{2} \psi_{1}$ and $\mathrm{d}_{2} \omega_{1}$ were subtracted from the MHB estimates of the integration constants to derive the corrected precession rates:

$$
\begin{aligned}
& r_{0}=\left(r_{0}\right)_{\mathrm{MHB}}-\mathrm{d}_{1} \psi_{1}-\mathrm{d}_{2} \psi_{1}=5038^{\prime \prime} .482040 \mathrm{cy}^{-1} \\
& u_{0}=\left(u_{0}\right)_{\mathrm{MHB}}-\mathrm{d}_{2} \omega_{1}=-0^{\prime \prime} .025754 \mathrm{cy}^{-1} .
\end{aligned}
$$

In our computation, the numerical integration is carried out using the above $r_{0}$ and $u_{0}$ as integration constants. Another way of dealing with $\mathrm{d}_{2} \psi_{1}$ and $\mathrm{d}_{2} \omega_{1}$ was suggested in the P04 paper (Capitaine et al. 2005), by which a new set of integration constants can be generated. The test of this problem with VLBI data is performed in Sect. 4.1.

\subsection{Polynomial expressions for the precession of the equator}

The upgraded precession of the equator is obtained by solving the differential Eqs. (10) by using (i) the updated ecliptic precession expressions derived from VSOP2013 and DE422 (cf. lines 1 and 3 in Table 3); (ii) the additional theoretical contributions to the precession rates described in Sect. 3.1; and (iii) integration constants $r_{0}$ and $u_{0}$ given in Eq. (19). The solutions for the basic precession quantities, $\psi_{\mathrm{A}}$ and $\omega_{\mathrm{A}}$, for the updated solution (denoted LC solution) are such that

$$
\begin{aligned}
\psi_{\mathrm{A}}= & 5038^{\prime \prime} .482040 t-1{ }^{\prime \prime} .07324 t^{2}+0^{\prime \prime} .01573401 t^{3} \\
& +00^{\prime \prime} 000127135 t^{4}-0 \prime \prime 0000001020 t^{5} \\
\omega_{\mathrm{A}}= & \epsilon_{0}-0^{\prime \prime} .025754 t+0^{\prime \prime} .0512625 t^{2}-0^{\prime \prime} .0077249 t^{3} \\
& -0^{\prime \prime} .000000267 t^{4}+0^{\prime \prime} .000000267 t^{5}
\end{aligned}
$$

with $\epsilon_{0}=84381$ "'406. The secondary quantities $p_{\mathrm{A}}, \epsilon_{\mathrm{A}}$, and $\chi_{\mathrm{A}}$ are simultaneously derived as:

$$
\begin{aligned}
p_{\mathrm{A}}= & 5028^{\prime \prime} .796891 t-1^{\prime \prime} .1111298 t^{2}+00^{\prime \prime} 01695523 t^{3} \\
& -00^{\prime \prime} 000020031 t^{4}-0^{\prime \prime} .000000017 t^{5} \\
\epsilon_{\mathrm{A}}= & \epsilon_{0}-46^{\prime \prime} .836735 t-0^{\prime \prime} .0001936 t^{2}+0^{\prime \prime} .00200005 t^{3} \\
& -0^{\prime \prime} .000000594 t^{4}+0^{\prime \prime} .000000012 t^{5} \\
\chi_{\mathrm{A}}= & 10^{\prime \prime} .556240 t-2^{\prime \prime} .3813876 t^{2}-0^{\prime \prime} 00121311 t^{3} \\
& +0^{\prime \prime} .000160286 t^{4}+0^{\prime \prime} .000000086 t^{5} .
\end{aligned}
$$

If the INPOP10a induced ecliptic precession (cf. lines 5 and 7 of Table 3 ) is used to replace the DE422 ecliptic in the integration, the resulting difference of $\psi_{\mathrm{A}}$ and $\omega_{\mathrm{A}}$ is (in $\mu$ as)

$$
\begin{aligned}
& \Delta \psi_{\mathrm{A}}=-0.003 t+0.9 t^{2}+0.02 t^{3} \\
& \Delta \omega_{\mathrm{A}}=-0.4 t^{2}+0.006 t^{3} .
\end{aligned}
$$

This confirms that the effect of different ecliptic precessions is completely negligible.

The comparison of the LC solution with the IAU 2006 precession is shown in Table 7. The largest difference in the quadratic and cubic terms for $\psi_{\mathrm{A}}$ and $p_{\mathrm{A}}$ is attributed to the use of updated empirical model for $J_{2}$ variation. The signs for the 
Table 7. Differences of the precession quantities between present solution LC and the IAU 2006 solution in the sense of $\Delta=$ [LC-IAU 2006].

\begin{tabular}{lcrrrr}
\hline \hline & $t^{1}$ & $t^{2}$ & $t^{3}$ & $t^{4}$ & $t^{5}$ \\
\hline$\Delta \psi_{\mathrm{A}}$ & 532 & 5765 & 16874 & -6 & -0.01 \\
$\Delta \omega_{\mathrm{A}}$ & -1 & 0.3 & 0.1 & 0.1 & -0.07 \\
$\Delta p_{\mathrm{A}}$ & 696 & 5695 & 16876 & 4 & 0.02 \\
$\Delta \epsilon_{\mathrm{A}}$ & 34 & -10 & -3 & -0.02 & 0.03 \\
$\Delta \chi_{\mathrm{A}}$ & -163 & 53 & -1 & -10 & -0.03 \\
\hline
\end{tabular}

Notes. Units of the coefficients: $\mu$ as, $t$ in Julian centuries from J2000.0.

$t^{3}$ terms of $\psi_{\mathrm{A}}$ are now positive while in IAU 2006 they are negative. The precession in obliquity $\omega_{\mathrm{A}}$ is identical with that of the IAU 2006 because the integration constant for both cases is the same: the only difference for $\omega_{\mathrm{A}}$ at the order smaller than $1 \mu \mathrm{as} \mathrm{cy}^{-1}$ originates from the $\epsilon$-dependence of these new theoretical contributions. The deviations in parameters $\epsilon_{\mathrm{A}}$ and $\chi_{\mathrm{A}}$ result from both new ecliptic and equator precessions. The largest uncertainties in our solution for the precession in longitude are still attributed to the imperfect modeling of the $J_{2}$ variation. Based on the fitted parameters in Table 5, the relative errors in linear and quadratic terms of $r_{\psi}$ are as high as $40 \%$ and $18 \%$ (see Table 6), responsible for about $0.5 \mathrm{mas} \mathrm{cy}^{-2}$ and $4.5 \mathrm{mas} \mathrm{cy}^{-3}$ uncertainties in the $t^{2}$ and $t^{3}$ terms of $\psi_{\mathrm{A}}$.

\section{Comparison of the precession expressions with VLBI celestial pole offsets}

The geodetic/astrometric VLBI technique plays a crucial role in understanding the Earth's rotation. It monitors the celestial coordinates of the CIP and the Universal Time (UT1), which are known as the Earth orientation parameters (EOP). The current accuracy of VLBI observations is unprecedentedly high, namely at microarcsecond level, thus it provides the best observational material for studying the behavior of the precession-nutation models. As VLBI observations have shown that there are deficiencies in the IAU 2006/2000 model of the order of 0.2 mas, mainly due to the fact that the free core nutation (FCN) is not part of the model, the IERS will continue to publish observed estimates of the corrections to the IAU 2006/2000 precessionnutation. The observed differences with respect to the IAUmodel-predicted CIP positions are reported as "celestial pole offsets" (CPO) $\mathrm{d} X$ and $\mathrm{d} Y$ :

$\mathrm{d} X_{\mathrm{IAU}}=X_{\mathrm{obs}}-X_{\mathrm{IAU}}, \quad \mathrm{d} Y_{\mathrm{IAU}}=Y_{\mathrm{obs}}-Y_{\mathrm{IAU}}$,

the subscript "IAU" meaning that the reference model is the standard IAU 2006/2000A $A_{\text {R06 }}$ precession-nutation model. Capitaine et al. (2009) applied several kinds of empirical models (linear, parabola, linear plus 18.6-yr nutation, and parabola plus 18.6-yr nutation) to fit the series of celestial pole offsets (19792008), in order to obtain the corrections for the IAU 2000 and IAU 2006/2000 precession-nutation models with respect to observations. In that work, there was an unexplained quadratic "curvature" in the residuals, which may be related to the Earth's $J_{2}$ rate uncertainty. We try to discuss this subject using the additional seven years of VLBI data (2009-2015) and the revised precession model LC.

To derive the celestial pole offsets with respect to the upgraded LC precession, we used the rigorous procedure as described in Capitaine (2006). First we evaluate the precession matrix $\mathcal{P}_{\mathrm{LC}}$ using the $\mathrm{LC}$ precession expressions:

$\mathcal{P}_{\mathrm{LC}}=\mathcal{R}_{3}\left(\chi_{\mathrm{A}}\right) \mathcal{R}_{1}\left(-\omega_{\mathrm{A}}\right) \mathcal{R}_{3}\left(-\psi_{\mathrm{A}}\right) \mathcal{R}_{1}\left(\epsilon_{0}\right)$,
Table 8. Weight mean (WM) and weighted mean root square (WRMS) of the celestial pole offsets related to the LC, LC' , and the IAU 2006 precession models.

\begin{tabular}{|c|c|c|c|c|c|}
\hline & & & IAU 2006 & $\mathrm{LC}$ & $\mathrm{LC}^{\prime}$ \\
\hline \multicolumn{2}{|l|}{ WM } & $\begin{array}{l}\mathrm{d} X \\
\mathrm{~d} Y\end{array}$ & $\begin{array}{r}0.047 \\
-0.057\end{array}$ & $\begin{array}{r}0.013 \\
-0.056\end{array}$ & $\begin{array}{r}0.030 \\
-0.117\end{array}$ \\
\hline \multicolumn{2}{|l|}{ Pre-fit WRMS } & $\begin{array}{l}\mathrm{d} X \\
\mathrm{~d} Y\end{array}$ & $\begin{array}{l}0.135 \\
0.144\end{array}$ & $\begin{array}{l}0.126 \\
0.144\end{array}$ & $\begin{array}{l}0.129 \\
0.178\end{array}$ \\
\hline \multirow{3}{*}{ Post-fit WRMS } & (i) & $\begin{array}{l}\mathrm{d} X \\
\mathrm{~d} Y\end{array}$ & $\begin{array}{l}0.125 \\
0.129\end{array}$ & $\begin{array}{l}0.125 \\
0.129\end{array}$ & \\
\hline & (ii) & $\begin{array}{l}\mathrm{d} X \\
\mathrm{~d} Y\end{array}$ & $\begin{array}{l}0.121 \\
0.124\end{array}$ & $\begin{array}{l}0.119 \\
0.124\end{array}$ & \\
\hline & (iii) & $\begin{array}{l}\mathrm{d} X \\
\mathrm{~d} Y\end{array}$ & $\begin{array}{l}0.124 \\
0.124\end{array}$ & $\begin{array}{l}0.118 \\
0.124\end{array}$ & \\
\hline
\end{tabular}

Notes. The unit is mas; (i); (ii); and (iii) refer to the three empirical models in Eq. (33).

where the parameters are given in Eqs. (20) and (21). This fourrotation transformation method makes use of the parameters that are derived directly from the dynamical solution and separates clearly the precession of the ecliptic and the equator. Then the nutation-precession-bias matrix $\mathcal{M}_{\mathrm{LC}}$ can be calculated using the IAU $2000 \mathrm{~A}_{\mathrm{R} 06}$ nutation matrix $\mathcal{N}$ and the frame bias matrix $\mathcal{B}$ at J2000.0:

$\mathcal{M}_{\mathrm{LC}}=\mathcal{N} \mathcal{P}_{\mathrm{LC}} \mathcal{B}$.

In the next step, the CIP coordinates corresponding the LC precession are extracted from the matrix $\mathcal{M}_{\mathrm{LC}}$ :

$X_{\mathrm{LC}}=\mathcal{M}_{\mathrm{LC}}(3,1) ; \quad Y_{\mathrm{LC}}=\mathcal{M}_{\mathrm{LC}}(3,2)$.

Finally, the theoretical predictions of $X_{\mathrm{LC}}$ and $Y_{\mathrm{LC}}$ are subtracted from the observed position to obtain the new celestial pole offsets:

$\mathrm{d} X_{\mathrm{LC}}=X_{\mathrm{obs}}-X_{\mathrm{LC}}, \quad \mathrm{d} Y_{\mathrm{LC}}=Y_{\mathrm{obs}}-Y_{\mathrm{LC}}$,

in which $X_{\mathrm{obs}}$ and $Y_{\mathrm{obs}}$ are derived from Eq. (23). The above calculations are carried out using the SOFA software package (Standard of Fundamental Astronomy, IAU SOFA board 2014).

The time series for celestial pole offsets ${ }^{3}$ are shown in Fig. 3 and refer to the LC and IAU 2006 precessions respectively. The free core nutation has been removed with the empirical model of the IERS (Petit \& Luzum 2010). Small curvatures can be seen from the time series of CPOs but the difference is not clearly distinguishable by the eye.

For each $\mathrm{d} X$ and $\mathrm{d} Y$ time series, we calculated the weighted mean (WM) value and the weighted root mean square (prefitWRMS) to indicate the overall consistency between the theoretical predictions and observations (see Table 8). Here the WRMS of an ensemble of data $x_{i}$ with standard errors $\sigma_{i}$ is defined as:

$\mathrm{WRMS}=\sqrt{\sum w_{i} x_{i}^{2} / \sum w_{i}}$,

where $w_{i}=1 / \sigma_{i}^{2}$ is the classical weight.

\subsection{Test the validity of P03-like and P04-like integration constants}

As mentioned in Sect. 3.2, spurious contributions to the MHB precession rates $d_{1} \psi_{1}, d_{2} \psi_{1}$, and $d_{2} \omega_{1}$ (cf. Eqs. (17) and (18)) 3 Solution derived at Paris Observatory, http://ivsopar.obspm.
$\mathrm{fr} /$ nutation/geo.dat 

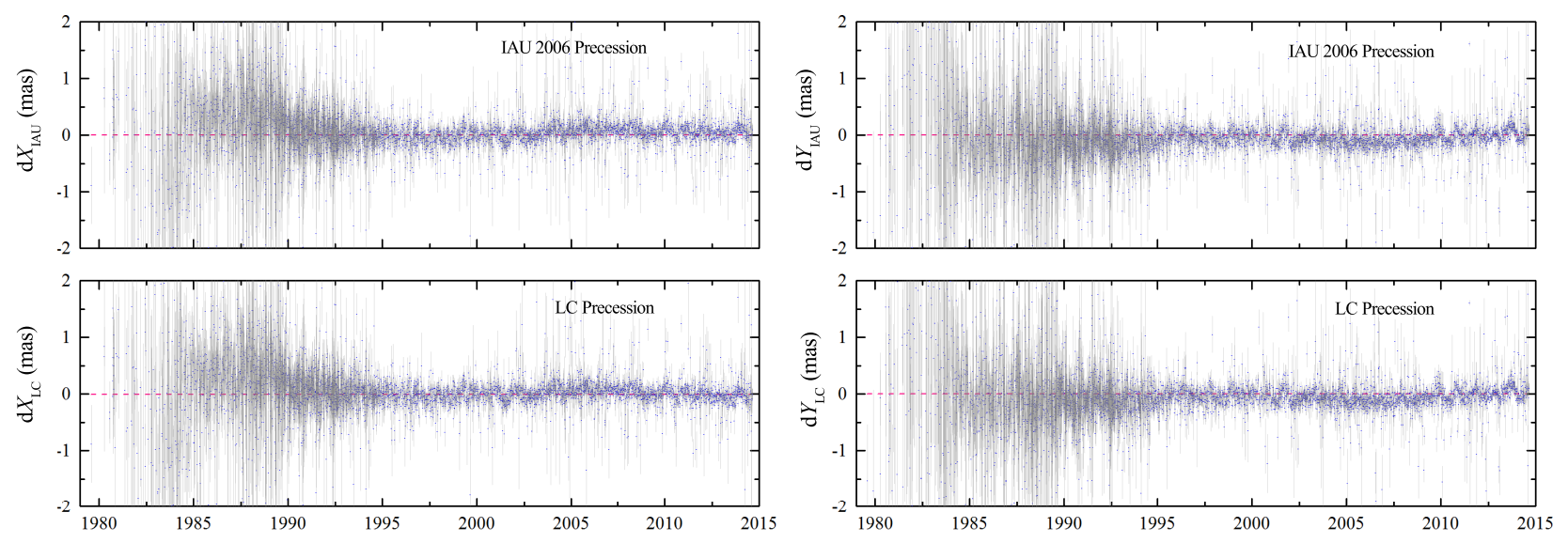

Fig. 3. Celestial pole offsets with respect to the LC and IAU 2006 precession expressions. The free core nutation (FCN) has been removed.

Table 9. Integration constants and main contributions from first order terms of luni-solar torques correspond to P03-like and P04-like ways.

\begin{tabular}{lccccccc}
\hline \hline & Solution & $\left(r_{0}\right)_{\mathrm{MHB}}$ & $r_{0}=\psi_{1}$ & $\left(r_{0}\right)_{1}$ & $\left(u_{0}\right)_{\mathrm{MHB}}$ & $u_{0}=\omega_{1}$ & $\left(u_{0}\right)_{1}$ \\
\hline \multirow{4}{*}{ P03-like } & & & $\left(r_{0}\right)_{\mathrm{MHB}}-\mathrm{d}_{1} \psi_{1}-\mathrm{d}_{2} \psi_{1}$ & $r_{0}-\left(r_{0}\right)_{\mathrm{th}}$ & & $\left(u_{0}\right)_{\mathrm{MHB}}-\mathrm{d}_{2} \omega_{1}$ & $u_{0}-\left(u_{0}\right)_{\mathrm{th}}$ \\
& LC & 5038.47929 & 5038.482040 & 5040.684190 & -0.02524 & -0.025754 & -0.028681 \\
& IAU 2006 & 5038.47875 & 5038.481507 & 5040.704677 & -0.026753 \\
\hline P04-like & & & $\left(r_{0}\right)_{\mathrm{MHB}}-\mathrm{d}_{1} \psi_{1}+\mathrm{d}_{2} \psi_{1}$ & $r_{0}-\left(r_{0}\right)_{\mathrm{th}}$ & & $\left(u_{0}\right)_{\mathrm{MHB}}+\mathrm{d}_{2} \omega_{1}$ & $u_{0}-\left(u_{0}\right)_{\mathrm{th}}$ \\
& LC $^{\prime}$ & 5038.47929 & 5038.481270 & 5040.683420 & -0.02524 & -0.024725 & -0.027625 \\
& P04 & & & 5040.724530 & -0.026064 \\
\hline
\end{tabular}

Notes. Units: arcsecond per century $\left("{ }^{\prime \prime} \mathrm{cy}^{-1}\right)$.

should be eliminated from $\left(r_{0}\right)_{\mathrm{MHB}}$ and $\left(u_{0}\right)_{\mathrm{MHB}}$. However, the way to apply the effect of $\mathrm{d}_{2} \psi_{1}$ and $\mathrm{d}_{2} \omega_{1}$ is controversial: the IAU 2006 (P03, Capitaine et al. 2003a) and later P04 model (Capitaine et al. 2005) applied these corrections with opposite signs. This caused a discrepancy of hundreds to one thousand microarcseconds per century in precession rates. We call the two conflicting approaches P03-like and P04-like. Our LC solution was computed using the P03-like method. We also tried to apply the P04-like integration constants as listed in Table 9 to derive a revised precession solution named $\mathrm{LC}^{\prime}$. All of the other details in the integration are exactly the same as in LC. According to the integration constants, the main contributions to precession rates $\left(r_{0}\right)_{1}$ and $\left(u_{0}\right)_{1}$ originate from the first order luni-solar terms and are also provided in this table, where $\left(r_{0}\right)_{\text {th }}$ and $\left(u_{0}\right)_{\text {th }}$ are the total theoretical contributions, for example, for the LC model, $\left(r_{0}\right)_{\mathrm{th}}=-2{ }^{\prime \prime} .202150 \mathrm{cy}^{-1}$ and for the IAU 2006, $\left(r_{0}\right)_{\mathrm{th}}=-2^{\prime \prime} \cdot 22317 \mathrm{cy}^{-1}$.

By integrating the precession equations, the primary parameters for the $\mathrm{LC}^{\prime}$ solution in longitude and obliquity are

$$
\begin{aligned}
\psi_{\mathrm{A}}= & 5038^{\prime \prime} .481270 t-1^{\prime \prime} .0732468 t^{2}+0^{\prime \prime} .01573403 t^{3} \\
& +0^{\prime \prime} .000127135 t^{4}-0^{\prime \prime} .0000001020 t^{5} \\
\omega_{\mathrm{A}}= & \epsilon_{0}-0^{\prime \prime} .024725 t+0^{\prime \prime} 0512626 t^{2}-0^{\prime \prime} .0077249 t^{3} \\
& -0^{\prime \prime} .000000267 t^{4}+0^{\prime \prime} .000000267 t^{5}
\end{aligned}
$$

with $\epsilon_{0}=84381$ '"406. Except for the linear terms, the difference larger than $1 \mu$ as between the $\mathrm{LC}$ and $\mathrm{LC}^{\prime}$ solutions only appears in the quadratic terms in longitude, and the differences in those terms can be written (in $\mu$ as) as:

$\Delta \psi_{\mathrm{A}}=770 t+6 t^{2} ; \quad \Delta \omega_{\mathrm{A}}=-1029 t$.

The VLBI time series corresponding to the $\mathrm{LC}^{\prime}$ precession are generated in the same way as for the LC solution, and the WM and WRMS values for this series are also presented in Table 8. This test can be presented as being an extension of the test of the P04 versus P03 solutions in Capitaine et al. (2005), with ten additional years of VLBI data. We note that the only difference between the LC and LC $^{\prime}$ solution is that different integration constants (P03-like and P04-like) have been used. For the $\mathrm{d} X$ component, the WM and WRMS for both LC and $\mathrm{LC}^{\prime}$ solutions are smaller than the IAU 2006 values. Regarding the $\mathrm{d} Y$ component, however, the WM and WRMS relative to the $\mathrm{LC}^{\prime}$ solution are even larger than the IAU model while the values relative to the LC solution are close to the IAU value, which means that the use $\mathrm{LC}^{\prime}$ precession solution deviates away from observations more than the other two models. In addition, we tried to integrate the precession equations with the IAU 2006 precession of the ecliptic and Earth model (theoretical contributions to the theoretical precession rates including $J_{2}$ rate) but with P04 interpretation of integration constants $r_{0}$ and $u_{0}$ was:

$$
\begin{aligned}
& \left(r_{0}\right)_{\mathrm{P} 04}=5038^{\prime \prime} .47875+0^{\prime \prime} .001982=5038^{\prime \prime} .480732 \mathrm{cy}^{-1} \\
& \left(u_{0}\right)_{\mathrm{P} 04}=-0^{\prime \prime} .02524+0^{\prime \prime} .000514=-00^{\prime \prime} 024726 \mathrm{cy}^{-1} .
\end{aligned}
$$

The resulting precession expressions are also checked with the same method and VLBI time series. The relative WM and WRMS of the celestial pole offsets are not surprisingly larger than the IAU 2006 values in Table 8:

$$
\begin{array}{ll}
\mathrm{WM}_{X}=0.0670 \text { mas; } & \mathrm{WRMS}_{X}=0.1480 \text { mas } \\
\mathrm{WM}_{Y}=-0.1168 \text { mas; } & \mathrm{WRMS}_{Y}=0.1795 \text { mas. }
\end{array}
$$

This confirms that the implementation of corrections $\mathrm{d}_{2} \psi_{1}$ and $\mathrm{d}_{2} \omega_{1}$ (Eq. (18)) to the integration constants should be identical to the P03/IAU 2006 model (P03-like approach). The above tests clearly allow us to conclude that the treatment of the integration constants in the LC solution is correct and that the P04-like case 
should be definitively eliminated. Therefore $\mathrm{LC}^{\prime}$ will not be considered in the rest of this paper. Only the LC solution is tested further.

\subsection{Comparison of the IAU 2006 versus $L C$ precession models with VLBI data}

First, we can see from Table 8 that the smallest WM and WRMS of the celestial pole offsets can be found when the LC precession model has been used to calculate the CIP location. For the $\mathrm{d} X$ component, the LC solution appears to be more consistent with VLBI observations than the current IAU 2006 precession in the global sense, since the WM decreases by about $72 \%$ with reference to the IAU one.

To interpret more thoroughly the residuals between the observations and the two precession solutions, we have used (i) a parabola; (ii) a straight line plus 18.6-yr nutation; and (iii) a parabola plus the 18.6-yr nutation as in Capitaine et al. (2009) for the least squares fit. The 18.6-yr nutation is the largest nutation term and is expected to be sensitive to the errors of the secular precession model. The equations used for the fit of the celestial pole offsets are such that:

$$
\mathrm{d} X=\left\{\begin{array}{r}
A_{0}+A_{1} t+A_{2} t^{2} \\
\mathrm{~d} Y \\
A_{0}+A_{1} t+A_{\mathrm{s}} \sin \Omega+A_{\mathrm{c}} \cos \Omega \\
A_{0}+A_{1} t+A_{2} t^{2}+A_{\mathrm{s}} \sin \Omega+A_{\mathrm{c}} \cos \Omega
\end{array}\right.
$$

where $\Omega$ (polynomial function of $t$ ) is the mean longitude of the ascending node of the Moon with a period of 6798.38 days (approximately equal to $18.6 \mathrm{yr})$.

The coefficients $\left(A_{0}, A_{1}, A_{2}, A_{\mathrm{s}}, A_{\mathrm{c}}\right)$ in the three functions of Eq. (33) are estimated from the weighted least squares fits and are listed in Table 10. It is clear that the longer time span of VLBI data reduced the coefficients of the quadratic model, especially the $t^{2}$ term, compared to the results in Capitaine et al. (2009), indicating that the IAU 2006 precession is effective for predicting the CIP position. Furthermore, the coefficient of $t^{2}$ term decreased significantly when the LC precession was adopted, but the signs are opposite to the IAU 2006 value. Since the most important change in the LC precession is the introduction of the updated $J_{2}$ variation, which mainly modifies the quadratic and cubic terms of the precession in longitude, we have shown that the use of the $J_{2}$ quadratic variation eliminated most of the residual quadratic curvature in the celestial pole offsets. This conclusion is consistent with previous checks by WM and WRMS. On the other hand, the resulting coefficients obtained from fitting linear plus 18.6 -yr or parabola plus 18.6 -yr periodic term differ significantly in each case. However, it is still difficult to discriminate which model is more appropriate to interpret the physical reason for the overall residuals because all of the post-fit WRMS in Table 8 reduced by approximately the same level. Another feature is the unstable $t^{1}$ coefficients for the LC model that may be due to the large $t^{3}$ term of $\psi_{\mathrm{A}}$ in the LC model (Eq. (29)).

Table 11 presents the correlation matrix of the coefficients in different empirical models. The elements in the upper triangular matrix correspond to the IAU 2006 precession while the low triangular matrix is for the LC solution. In the parabola model, the constant-quadratic and linear-quadratic correlation coefficients are up 0.7 for both precession models, showing that the VLBI residuals cannot be simply interpreted by quadratic function. This conclusion is similar to Capitaine et al. (2009). In the model comprising of the linear plus $18.6 \mathrm{yr}$ periodic term, the correlation coefficients between linear and sine term for the IAU model is as high as 0.5 , but it decreases to about 0.2 in the case of the
LC precession. From Table 11 we can conclude that the linear plus $18.6 \mathrm{yr}$ is better than the other two empirical models especially for the LC precession. For the parabola plus $18.6 \mathrm{yr}$ periodic term, which was abandoned by Capitaine et al. (2009), the correlation coefficients for the updated LC model (mean absolute value of 0.19 ) are globally lower than the IAU model (mean absolute value of 0.42 ) except for the 0.7 correlation between $A_{0}$ and $A_{2}$. Indeed, the fit using the last empirical model leads to the reasonable inference that the quadric and period behaviors in the VLBI residuals become noticeable and separable with longer VLBI time series and the updated long-term variation of $J_{2}$. The unexplained "curvature" in the residuals in previous studies (quite difficult to interpret in the past) may be expected to be modeled by a more realistic Earth model.

For the $\mathrm{d} Y$ component, the fitted results (see Tables 10 and 11) are identical for the LC and the IAU model since the expressions for $\omega_{\mathrm{A}}$ are almost the same. The fitted coefficients reduced significantly with respect to Capitaine et al. (2009) thanks to the longer time span of the VLBI data.

\section{Summary and discussion}

In this work we have investigated the possibility of improving the IAU 2006 precession (Capitaine et al. 2003a) model with progress in the latest decade and an approach similar to the IAU 2006 method. The precession of the ecliptic has been derived using the latest analytical ephemerides VSOP2013 and numerical ephemerides DE422/INPOP10a. The polynomials for $p$ and $q$ provided by VSOP2013 are improved by fitting them to the DE422 and INPOP10a over 2000 yr centered on J2000.0. The resulting $P_{\mathrm{A}}$ and $Q_{\mathrm{A}}$ differ from the IAU 2006 parameters by several tens of microarcseconds per century in secular terms, but this can be considered as negligible for the calculation of the precession of the equator. The differences in higher order terms are also too small to be considered.

The precession of the equator was obtained by solving the dynamical equations taking into account the revised precession of the ecliptic, the new theoretical contributions to the precession rates, and the updated integration constants. The progress in theoretical precession rates includes contributions from revised non-linear terms, tidal Poisson terms, second-order torque, Galactic aberration, and the most important new determination of the $J_{2}$ variation. Based on the 35-year monitoring by the SLR, the long-term variations $J_{2}$ appears to be more quadratic than linear in nature. This feature modified the quadratic and cubic terms in the precession in longitude $\psi_{\mathrm{A}}$ by about $5700 \mu \mathrm{as} \mathrm{cy}^{-2}$ and $16800 \mu \mathrm{as} \mathrm{cy}^{-3}$, respectively. For the integration constants, we checked two interpretation of the correction from the nonrigorous treatment of bias and found that the approach used in the IAU 2006 model is correct: the P04-like approach should be eliminated. The LC solution is therefore recommended as the upgraded expression for the precession of the equator, but the accuracy of the updated precession is mainly restricted by the uncertainties in the $J_{2}$ empirical model.

We have compared our solution to the IAU 2006 model with the most accurate VLBI series of celestial pole offsets. The weight mean and weighted root mean square values of the $\mathrm{d} X$ celestial pole offsets relative to the LC precession reduced by about $72 \%$ and $7 \%$. The fits of different empirical models to the longer VLBI residuals have shown the different characteristics of the LC and IAU 2006 precession, mainly due to the adoption of a new $J_{2}$ variation. The improvement in correlation coefficients implies that the linear plus $18.6 \mathrm{yr}$ term is the most efficient. The model with parabola plus $18.6 \mathrm{yr}$ term begins to be effective as 
Table 10. Weighted fits of empirical models (Eq. (33)) to the VLBI residuals corresponding to the LC and IAU 2006 precession.

\begin{tabular}{|c|c|c|c|c|c|}
\hline \multirow{2}{*}{ Empirical model } & \multirow{2}{*}{ Coefficient } & \multicolumn{2}{|c|}{ IAU 2006 model } & \multicolumn{2}{|c|}{ LC model } \\
\hline & & $\mathrm{d} X$ & $\mathrm{~d} Y$ & $\mathrm{~d} X$ & $\mathrm{~d} Y$ \\
\hline (i) Quadratic & $\begin{array}{l}A_{0} \\
A_{1} \\
A_{2}\end{array}$ & $\begin{array}{l}+0.200 \pm 0.003 \\
+0.152 \pm 0.039 \\
+2.423 \pm 0.387\end{array}$ & $\begin{array}{l}-0.093 \pm 0.003 \\
+0.081 \pm 0.040 \\
+4.239 \pm 0.405\end{array}$ & $\begin{array}{l}+0.022 \pm 0.003 \\
-0.111 \pm 0.039 \\
-0.368 \pm 0.389\end{array}$ & $\begin{array}{l}-0.093 \pm 0.003 \\
+0.082 \pm 0.041 \\
+4.246 \pm 0.405 \\
\end{array}$ \\
\hline (ii) Linear $+18.6 \mathrm{yr}$ & $\begin{array}{l}A_{0} \\
A_{1} \\
A_{\mathrm{s}} \\
A_{\mathrm{c}}\end{array}$ & $\begin{array}{l}+0.028 \pm 0.002 \\
+0.188 \pm 0.029 \\
+0.028 \pm 0.003 \\
-0.046 \pm 0.003\end{array}$ & $\begin{array}{l}-0.079 \pm 0.002 \\
+0.475 \pm 0.030 \\
+0.007 \pm 0.003 \\
-0.061 \pm 0.003\end{array}$ & $\begin{array}{l}+0.204 \pm 0.002 \\
-0.230 \pm 0.029 \\
+0.041 \pm 0.002 \\
-0.042 \pm 0.003\end{array}$ & $\begin{array}{l}-0.079 \pm 0.002 \\
+0.477 \pm 0.030 \\
+0.007 \pm 0.003 \\
-0.061 \pm 0.003\end{array}$ \\
\hline (iii) Quadratic + $18.6 \mathrm{yr}$ & $\begin{array}{l}A_{0} \\
A_{1} \\
A_{2} \\
A_{\mathrm{s}} \\
A_{\mathrm{c}}\end{array}$ & $\begin{array}{l}-0.006 \pm 0.003 \\
-0.263 \pm 0.041 \\
+8.428 \pm 0.544 \\
+0.014 \pm 0.003 \\
+0.067 \pm 0.003\end{array}$ & $\begin{array}{l}-0.084 \pm 0.003 \\
+0.395 \pm 0.044 \\
+1.473 \pm 0.580 \\
+0.136 \pm 0.004 \\
-0.576 \pm 0.003\end{array}$ & $\begin{array}{l}+0.030 \pm 0.053 \\
-0.194 \pm 0.039 \\
-3.783 \pm 0.471 \\
-0.072 \pm 0.050 \\
+0.067 \pm 0.052\end{array}$ & $\begin{array}{l}-0.084 \pm 0.003 \\
+0.397 \pm 0.044 \\
+1.483 \pm 0.580 \\
+0.136 \pm 0.004 \\
-0.576 \pm 0.003\end{array}$ \\
\hline
\end{tabular}

Notes. The unit is mas.

Table 11. Correlation matrix in the least squares fit for different empirical models.

\begin{tabular}{|c|c|c|c|c|c|c|c|c|c|c|c|}
\hline \multirow{2}{*}{ Empirical model } & & \multicolumn{5}{|c|}{$\mathrm{d} X$ component } & \multicolumn{5}{|c|}{$\mathrm{d} Y$ component } \\
\hline & & $A_{0}$ & $A_{1}$ & $A_{2}$ & $A_{\mathrm{s}}$ & $A_{\mathrm{c}}$ & $A_{0}$ & $A_{1}$ & $A_{2}$ & $A_{\mathrm{s}}$ & $A_{\mathrm{c}}$ \\
\hline \multirow{3}{*}{ (i) Quadratic } & $A_{0}$ & 1 & -0.1 & -0.5 & & & 1 & -0.1 & -0.5 & & \\
\hline & $A_{1}$ & -0.1 & 1 & -0.7 & & & & 1 & -0.7 & & \\
\hline & $A_{2}$ & -0.7 & +0.3 & 1 & & & & & 1 & & \\
\hline \multirow{4}{*}{ (ii) Linear $+18.6 \mathrm{yr}$} & $A_{0}$ & 1 & -0.6 & & -0.1 & -0.0 & 1 & -0.6 & & -0.1 & +0.0 \\
\hline & $A_{1}$ & +0.2 & 1 & & +0.5 & -0.2 & & 1 & & +0.5 & -0.2 \\
\hline & $A_{\mathrm{s}}$ & +0.3 & +0.2 & & 1 & -0.2 & & & & 1 & -0.2 \\
\hline & $A_{\mathrm{c}}$ & -0.2 & +0.2 & & -0.1 & 1 & & & & & 1 \\
\hline \multirow{5}{*}{ (iii) Quadratic + $18.6 \mathrm{yr}$} & $A_{0}$ & 1 & +0.1 & -0.7 & -0.5 & -0.3 & 1 & +0.1 & -0.6 & -0.5 & -0.3 \\
\hline & $A_{1}$ & -0.1 & 1 & -0.7 & -0.3 & -0.4 & & 1 & -0.7 & -0.2 & -0.4 \\
\hline & $A_{2}$ & -0.7 & +0.3 & 1 & +0.7 & +0.4 & & & 1 & +0.6 & +0.4 \\
\hline & $A_{\mathrm{s}}$ & +0.2 & +0.2 & +0.1 & 1 & +0.1 & & & & 1 & +0.2 \\
\hline & $A_{\mathrm{c}}$ & -0.1 & +0.1 & -0.1 & -0.0 & 1 & & & & & 1 \\
\hline
\end{tabular}

Notes. For the $\mathrm{d} X$ components, the right upper triangular and left lower triangular elements correspond to the IAU 2006 and LC precessions, respectively. For the $\mathrm{d} Y$ component, the correlation coefficients are equal for the LC and IAU models.

more VLBI data are available, and it would be helpful for us to understand the long periodic signals in the VLBI residuals.

\subsection{Choice of the standard precession model}

The LC precession solution developed in this paper is based on recent improvements in EMB motion and theoretical contributions to precession. Our solution refined the IAU standard model to a certain extent and has potential to replace the IAU 2006 precession model in the future. However, at present, we recommend the continued use of the current IAU model for the following reasons: (i) the changes in the precession of the ecliptic can be considered as negligible; (ii) the $J_{2}$ variation (which is the most important feature of the LC solution) can still be approximated by an empirical model with relatively large uncertainties but cannot be predicted by geophysical theories. In Cheng et al. (2013), new models for the $J_{2}$ variations have been proposed but no model for the secular term is considered as being adopted by the geophysical community. Moreover, it is not possible to compare the validity of the corresponding quadratic terms in precession using only $35 \mathrm{yr}$ of VLBI observations. An expression for $\dot{j}_{2}$ has been proposed in this paper but this cannot be considered as providing a more accurate expression yet; (iii) improvement of the
LC solution is not very convincing from the comparison with VLBI; (iv) the precession model itself is a secular phenomenon over tens of thousand years: ten years of progress seems insufficient to change the standard of the model; (v) the IAU 2006 precession is widely used by researchers in fundamental astronomy, especially in VLBI data reduction, and frequent change of the precession is not helpful for revealing the physical reason for its deficiency.

\subsection{The role of the ecliptic and the future precession-nutation model of the Earth}

After the adoption by the IAU of the International Celestial Reference System (ICRS) and new CIO-based parameterization for describing the Earth's rotation, the problems concerning the role of the ecliptic in modern fundamental astronomy was proposed (Capitaine \& Soffel 2015). To develop the precession of the equator, the conventional ecliptic was usually introduced as a reference plane to help the authors to write the simplified differential equations and apply the semi-analytical approach with precession rates provided by theories and observations. However, it should be remembered that the precession-nutation models describe the position of the Earth's rotation pole (CIP) rather 
than the ecliptic pole in space, so that the ecliptic can, in principle, be abandoned in Earth rotation studies. For example, the CIO-based parameterization can be written without any concept of the ecliptic. We also note that the use of the ecliptic is not mandatory since the dynamical equations for the Earth's rotation can be developed using directly the CIP coordinates $X$ and $Y$ (e.g., Capitaine et al. 2006), where the ecliptic was avoided. This subject will be discussed more deeply but it is clear that the role of the ecliptic will not be as crucial as it was in the past.

As for the future of the theory of the Earth's rotation, the secular part, namely precession, and the periodic part, namely nutation, should be solved simultaneously to provide a selfconsistent model (see e.g., Escapa et al. 2014). The VLBI will still be the main observational technique to refine the model of the Earth's rotation. Furthermore, the use of the Gaia (Perryman et al. 2001) reference system will bring an opportunity to compare the precession-nutation in optical bandpass with that determined by VLBI at a homogeneous level of accuracy which could not be done in the past.

Acknowledgements. This work is funded by the National Natural Science Foundation of China (NSFC) under grant Nos. 11303018 and 11473013 and the Natural Science Foundation of Jiangsu Province under No. BK20130546. The authors are grateful to the referee of this paper for his/her valuable suggestions to improve the text.

\section{References}

Bretagnon, P., \& Francou, G. 1988, A\&A, 202, 309

Bretagnon, P., Rocher, P., \& Simon, J.-L. 1998, A\&A, 329, 329

Bretagnon, P., Fienga, A., \& Simon, J.-L. 2003, A\&A, 400, 785

Bourda, G., \& Capitaine, N. 2004, A\&A, 428, 691

Capitaine, N. 2012, Proc. Journées 2011, Systèmes de Référence spatiotemporels, eds. H. Schuh, S. Bohm, T. Nilsson, \& N. Capitaine, Vienna University of Technology, 266

Capitaine, N., \& Soffel, M. 2015, Proc. Journées 2014, Systèmes de Référence spatio-temporels, eds. Z. Malki, \& N. Capitaine, Pulkovo observatory, 61

Capitaine, N., \& Wallace, P. T. 2006, A\&A, 450, 855

Capitaine, N., Wallace, P. T., \& Chapront, J. 2003a, A\&A, 412, 567 (IAU 2006/P03)

Capitaine, N., Chapront, J., Lambert, S., \& Wallace, P. T. 2003b, A\&A, 400, 1145
Capitaine, N., Wallace, P. T., \& Chapront, J. 2004, A\&A, 421, 365 Capitaine, N., Wallace, P. T., \& Chapront, J. 2005, A\&A, 432, 355 (P04)

Capitaine, N., Folgueira, M., \& Souchay, J. 2006, A\&A, 445, 347

Capitaine, N., Mathews, P. M., Dehant, V., Wallace, P. T., \& Lambert, S. B. 2009, CeMDA, 103, 179

Cheng, M. K., \& Tapley, B. D. 2004, J. Geophys. Res., 109, B009402

Cheng, M. K., Tapley, B. D., \& Ries, J. C. 2013, J. Geophys. Res., 118, 740

Escapa, A., Getino, J., Ferrándiz, J. M., \& Baenas, T. 2014, Proc. the Journées 2013, Systèmes de référence spatio-temporels, ed. N. Capitaine, Observatoire de Paris, 148

Fienga, A., Manche, H., Kuchynka, P., et al. 2010, ArXiv e-prints [arXiv: 1011.4419v1]

Fienga, A., Laskar, J., Kuchynka, P., et al., 2011, Celest. Mech. Dyn. Astr., 111, 363

Folgueira, M., Dehant, V., Lambert, S. B., \& Rambaux, N. 2007, A\&A, 469, 1197

Folkner, W. M., Williams, J. G., \& Boggs, D. H. 2008, JPL Memorandum IOM 343R-08-003

Fukushima, T. 2003, AJ, 126, 494

Gerlach, E., Klioner, S., \& Soffel, M. 2012, ArXiv e-prints [arXiv: $1202.5870 \mathrm{v} 1]$

Harada, W., \& Fukushima, T. 2004, AJ, 127, 531

Herring, T. A., Mathews, P. M., \& Buffett, B. A. 2002, J. Geophys. Res., 107, 4

Hilton, J. L., Capitaine, N., Chapront, J., et al. 2006, Celest. Mech. Dyn. Astron., 94, 351

IAU SOFA Board, IAU SOFA Software Collection, Issue 2013-12-02, available at http: //www.iausofa.org

Lambert, S. B., \& Mathews, P. M. 2006, A\&A, 453, 363

Lambert, S. B., \& Mathews, P. M. 2008, A\&A, 481, 883

Lieske, J. H., Lederle, T., Fricke, W., \& Morando, B. 1977, A\&A, 58, 1 (IAU 1976)

Liu, J.-C., Capitaine, N., Lambert, S. B., Malkin, Z., \& Zhu, Z. 2012, A\&A, 548, A50

Luzum, B., Capitaine, N., Fienga, A., et al. 2011, Celest. Mech. Dyn. Astron., 110,293

Mathews, P. M., Herring, T. A., \& Buffett, B. A. 2002, J. Geophys. Res., 107, 4 Nerem, R. S., Chao, B. F., Au, A. Y., et al. 1993, Geophys. Res. Lett., 20, 595

Perryman, M. A. C., de Boer, K. S., Gilmore, G., et al. 2001, A\&A, 369, 339

Petit, G., \& Luzum, B. 2010, IERS Conventions (2010), IERS Technical Note No. 36

Simon, J.-L., Francou, G., Fienga, A., \& Manche, H. 2013, A\&A, 557, A49

Standish, E. 1998, JPL Interoffice Memorandum, 312, 98

Stephenson, E. R., \& Morrison, L. V. 1995, Philos. Trans. R. Soc. London, Ser. A, 351, 165

Williams, J. G. 1994, AJ, 108, 711

Yoder, C. F., Williams, J.-G., Dickey, O., et al. 1983, Nature, 303, 757 